\title{
Seasonal Incidence of Raoiella indica Hirst and its Natural Enemies on Arecanut
}

\author{
Indhusri Chavan*, S. Pradeep, M. Manjunatha, H. Narayanaswamy and S. Sridhara
}

UAHS, Shivamogga, India

*Corresponding author

\section{Keywords}

Seasonal incidence, natural enemies, arecanut, Raoiella indica, correlation.

Article Info

Accepted:

18 January 2020

Available Online:

10 February 2020

A B S T R A C T

Study on seasonal incidence on Raoiella indica Hirst and its natural enemies were carried out at Sominkoppa, Shivamogga taluk during May 2016 to April 2018. The pooled analysis revealed that, the $R$. indica population was highest during first fortnight of March $\left(39.42 / \mathrm{cm}^{2}\right.$ of leaflet), while the lowest mites of $1.69 / \mathrm{cm}^{2}$ of leaflet were recorded during first fortnight of November. The natural enemies population viz., Stethorous keralicus and Scolothrips spp was highest during first fortnight of march coinciding with peak density of $R$. indica. Whereas, the predatory mite, A. largoensis was highest in the month of January, May and November months. Correlation studies revealed a significant positive association with maximum temperature $\left(\mathrm{r}=0.636^{* *}\right)$ while negative nonsignificant correlation with morning relative humidity $\left(\mathrm{r}=-0.700^{* *}\right)$, evening relative humidity $\left(r=-0.468^{*}\right)$ and Rainfall $(r=-0.306)$.

\section{Introduction}

Areca catechu L. is commonly known as arecanut or betel nut is a very widely cultivated plant in Eastern countries like India, Bangladesh, Sri Lanka, Malaysia, the Philippines and Japan. The importance of this nut is due to its use for chewing purposes. It is a common masticatory nut, consumed by all sections of the population, cutting across caste, class, region, religion, age and gender in India. Arecanut is an important cash crop forms a major source of income along with other agricultural crops. However, the farmers encountered considerable crop losses intermittently, owing to lack of knowledge on the bionomics, relative distribution pattern and ecological aspects of the pests.

Arecanut is attacked by an array of insect and non-insect pests. The pests infest all parts of the palm viz., stem, leaves, inflorescence, root and nuts. As many as 102 insect and noninsect pests have been reported to be 
associated with arecanut palm (Nair and Daniel, 1982), among which Raoiella indica is serious pest, which feeds on the underside of palm fronds of various hosts in the orders Arecales and Zingiberales. It was reported as a serious pest of economically important fruitproducing trees like the coconut, Cocos nucifera and banana, Musa spp (Nagesha Chandra and Channabasavanna, 1984; Welbourn, 2006) and it formed the first mite species in which feeding was observed through the stomata of its host plants (Ochoa et al., 2011). Through this specialized feeding habit, $R$. indica interfere with the photosynthesis and respiration processes of its host plants. Mite infested palms displayed stunted growth and withering of leaves.

\section{Materials and Methods}

The study on seasonal incidence of Raoiella indica and its natural enemies was carried out in Shivamogga taluk from May 2016 to April 2018. For this study areca garden consisting of four to five years old palm was selected and in each garden, five palms were selected randomly of which three leaflets are selected each from top, middle and basal fronds. Mites were counted in square $\mathrm{cm}$ area using hand lens (10X) at fortnightly intervals. The incidence of natural enemies viz., predatory coccinellids, thrips and phytoseiid mites were recorded as per leaflet and correlated with weather parameters viz., temperature, humidity and rainfall.

\section{Results and Discussion}

\section{During 2016-17}

The Raoiella indica occurred throughout the study period (May 2016 to April 2017), the population varied from 1.78 to 37.03 mites $/ \mathrm{cm}^{2}$ of leaflet (Table 1) and there was a gradual increase in mite population from the second fortnight of December with the rise in environmental temperature and continued to reach its peak during February second fortnight. The mite population reduced gradually in subsequent months with the fall in temperature, increased relative humidity and rainfall.

Seasonal incidence of natural enemies indicated that the predatory coccinellid, Stethorus keralicus (Kapur) and predatory thrips, Scolothrips spp. maintained a very thin population at the initial stage of mite infestation but the population build up acquired a high momentum with rolling on months (Table 1). The peak population of $S$. keralicus (16.60/leaflet) and Scolothrips spp. (9.32/leaflet) were found during second fortnight of February coinciding with those of their host.

The S. keralicus and Scolothrips spp. the population showed a decrease in trend with reduction of mite population in the months of September, October, November and December. Whereas, the predatory mite, Ambylesius largoensis population was very low (1.08/leaflet) during peak incidence period of mites, while it attained to maximum density during January, May and November month.

Correlation studies of mite with weather parameter revealed that maximum temperature $\left(\mathrm{r}=0.498^{* *}\right)$ and evening relative humidity $(\mathrm{r}=0.528 * *)$ had highly significant, positive association (Table 4) while minimum temperature $(\mathrm{r}=-0.007)$, morning relative humidity $(\mathrm{r}=-0.330)$ and rainfall $(\mathrm{r}=-0.06)$ were negatively correlated.

Predatory coccinellid S. keralicus has exhibited, a Positive correlation with maximum temperature $(\mathrm{r}=0.519 * *)$ and minimum temperature $(\mathrm{r}=0.088)$, while evening relative humidity showed highly significant negative relationship $(\mathrm{r}=-$ $\left.0.568^{* *}\right)$. The morning relative humidity $(\mathrm{r}=-$ 
0.261) and rainfall ( $\mathrm{r}=\quad-0.019)$ were negatively correlated (Table 4 ). There was a highly significant positive association observed between Scolothrips spp. with evening relative humidity $\left(\mathrm{r}=0.057^{*} *\right)$ and maximum temperature $\left(\mathrm{r}=0.505^{*}\right)$. While rainfall $(\mathrm{r}=-0.115)$, minimum temperature $(\mathrm{r}=$ $-0.022)$ and morning relative humidity $(\mathrm{r}=$ 0.266) were negatively correlated (Table 4). A. largoensis showed positive correlation with maximum temperature $(\mathrm{r}=0.342)$, while minimum temperature $(\mathrm{r}=-0.141)$, morning relative humidity $(\mathrm{r}=-0.359)$, evening relative humidity $(\mathrm{r}=-0.155)$ and rainfall $(\mathrm{r}=-0.133)$ had negative relationship.

\section{During 2017-18}

$R$. indica mite population remained active around the year and gradually increased during various months of the year (Table 2). It increased at a faster rate from the first fortnight of March $\left(46.87 / \mathrm{cm}^{2}\right.$ of leaflet). The population has started to decline in the following months with changes in environmental conditions. The natural enemies viz., Scolothrips spp. and $S$. keralicus population was highest during first fortnight of March (12.34 and 16.80 per leaflet) coinciding with the maximum incidence period of mites (Table 2). Lowest counts of $S$. keralicus (0.28 / leaflet) and Scolothrips spp. (0.10/leaflet) were recorded in the month of December. The A. largoensis was lowest during first and second fortnight $(0.06$ and $0.08 /$ leaflet respectively) of August and during peak mite incidence period it was 1.92 per leaflet.

Correlation study revealed that there was a highly significant positive relationship noticed between maximum temperature $(\mathrm{r}=$ $\left.0.775^{* *}\right)$ and mite population, whereas minimum temperature $(r=0.055)$ had a positive non-significant association (Table 4). Morning relative humidity $\left(\mathrm{r}=-0.777^{* *}\right)$ and rainfall $(\mathrm{r}=-0.306)$ were negatively correlated.

S. keralicus had positive, highly significant correlation with maximum temperature $(\mathrm{r}=$ $0.721 * *)$ whereas, minimum temperature $(\mathrm{r}=$ 0.183) showed positive non-significant association. The morning relative humidity $(\mathrm{r}=-0.703 * *)$ and evening relative humidity $(\mathrm{r}=-0.684 * *)$ had highly significant negative correlation. While rainfall $(\mathrm{r}=-0.166)$ exhibited non-significant negative relationship. Similarly, Scolothrips spp. had highly significant negative association with morning $\left(\mathrm{r}=-0.761^{* *}\right)$ and evening $(\mathrm{r}=$ $0.740 * *)$ relative humidity. While, rainfall $(\mathrm{r}=$ -0.208 ) is negatively correlated (Table 4). There was positive non-significant relationship observed with minimum temperature $(r=-0.100)$.

From all the above results it was evident that abiotic factors were found to exert a profound influence in determining the population size of $R$. indica. Increase in the temperature along with fairly low relative humidity enhanced the mite population. Similarly, $R$. indica population declined in an inconsistent manner as the atmospheric relative humidity increased.

\section{Pooled data}

Pooled data indicated that the $R$. indica persisted on arecanut palm throughout the year with notable fluctuations in the population, which varied from 1.11 to 39.42 mites per $\mathrm{cm}^{2}$ of leaflet (Table 3). Highest mites counts were recorded during first fortnight of March (39.42 / $\mathrm{cm}^{2}$ of leaflet), while the lowest mites of $1.69 / \mathrm{cm}^{2}$ of leaflet were recorded during first fortnight of November. The natural enemies viz., Scolothrips spp. and S. keralicus population was highest during first fortnight of March (12.91 and 17.89 per leaflet) coinciding with the maximum incidence of mites. 
Table.1 Seasonal incidence of Raoiella indica and its natural enemies on areca palms in Sominakoppa, Shivamogga taluk during 2016-17

\begin{tabular}{|c|c|c|c|c|c|c|c|c|}
\hline \multirow[t]{2}{*}{ Month / Year } & \multirow[t]{2}{*}{ Fortnight } & \multicolumn{4}{|c|}{ Mean mite population per $\mathrm{cm}^{2}$ of leaflet ${ }^{\#}$} & \multicolumn{3}{|c|}{ Natural enemies per leaflet } \\
\hline & & Top & Middle & Bottom & Mean & $\begin{array}{c}\text { Stethorus } \\
\text { keralicus }\end{array}$ & Scolothrips spp & $\begin{array}{c}\text { Amblyseius } \\
\text { largoensis }\end{array}$ \\
\hline \multirow[t]{2}{*}{ May 2016} & I & $14.82 \pm 2.02$ & $16.95 \pm 1.23$ & $20.70 \pm 1.68$ & $17.49 \pm 1.71$ & $9.50 \pm 0.46$ & $6.48 \pm 0.31$ & $4.30 \pm 0.21$ \\
\hline & II & $15.24 \pm 1.20$ & $16.75 \pm 1.04$ & $20.07 \pm 1.34$ & $17.35 \pm 1.42$ & $7.64 \pm 0.37$ & $5.45 \pm 0.26$ & $4.68 \pm 0.23$ \\
\hline \multirow[t]{2}{*}{ June } & I & $14.36 \pm 0.96$ & $13.13 \pm 0.94$ & $19.36 \pm 0.82$ & $15.62 \pm 1.90$ & $6.96 \pm 0.34$ & $5.56 \pm 0.27$ & $1.36 \pm 0.07$ \\
\hline & II & $9.65 \pm 0.55$ & $11.70 \pm 077$ & $14.11 \pm 0.59$ & $11.82 \pm 1.28$ & $5.89 \pm 0.28$ & $3.46 \pm 0.17$ & $1.78 \pm 0.09$ \\
\hline \multirow[t]{2}{*}{ July } & I & $5.04 \pm 0.56$ & $5.54 \pm 0.35$ & $10.70 \pm 0.44$ & $7.09 \pm 1.80$ & $5.02 \pm 0.24$ & $2.58 \pm 0.12$ & $1.08 \pm 0.05$ \\
\hline & II & $4.73 \pm 0.57$ & $6.40 \pm 0.59$ & $6.88 \pm 0.63$ & $6.00 \pm 0.65$ & $4.56 \pm 0.22$ & $3.59 \pm 0.17$ & $0.32 \pm 0.02$ \\
\hline \multirow[t]{2}{*}{ August } & I & $4.40 \pm 0.93$ & $5.44 \pm 0.26$ & $5.52 \pm 0.39$ & $5.12 \pm 0.36$ & $3.76 \pm 0.18$ & $3.47 \pm 0.17$ & $0.06 \pm 0.00$ \\
\hline & II & $4.41 \pm 0.44$ & $5.36 \pm 0.36$ & $6.76 \pm 0.42$ & $5.51 \pm 0.68$ & $4.06 \pm 0.20$ & $2.59 \pm 0.12$ & $0.04 \pm 0.00$ \\
\hline \multirow[t]{2}{*}{ September } & I & $2.47 \pm 0.55$ & $2.35 \pm 0.48$ & $2.57 \pm 0.39$ & $2.46 \pm 0.06$ & $3.21 \pm 015$ & $2.34 \pm 0.11$ & $0.20 \pm 0.01$ \\
\hline & II & $1.76 \pm 0.31$ & $1.64 \pm 0.19$ & $1.93 \pm 0.31$ & $1.78 \pm 0.08$ & $2.73 \pm 0.13$ & $1.98 \pm 0.10$ & $0.30 \pm 0.01$ \\
\hline \multirow[t]{2}{*}{ October } & I & $0.32 \pm 0.10$ & $6.96 \pm 0.45$ & $7.79 \pm 0.67$ & $4.92 \pm 2.36$ & $4.60 \pm 0.22$ & $1.03 \pm 0.05$ & $1.09 \pm 0.05$ \\
\hline & II & $3.21 \pm 0.74$ & $4.68 \pm 0.41$ & $6.89 \pm 0.80$ & $4.93 \pm 1.06$ & $5.08 \pm 0.24$ & $0.84 \pm 0.04$ & $2.28 \pm 0.11$ \\
\hline \multirow[t]{2}{*}{ November } & I & $1.58 \pm 0.20$ & $3.68 \pm 0.27$ & $4.28 \pm 0.51$ & $3.24 \pm 0.81$ & $2.52 \pm 0.12$ & $0.74 \pm 0.04$ & $3.06 \pm 0.15$ \\
\hline & II & $1.08 \pm 0.12$ & $1.92 \pm 0.26$ & $3.42 \pm 0.32$ & $2.14 \pm 0.68$ & $3.36 \pm 0.16$ & $0.32 \pm 0.02$ & $3.80 \pm 0.18$ \\
\hline \multirow[t]{2}{*}{ December } & I & $0.40 \pm 0.17$ & $1.03 \pm 0.25$ & $6.13 \pm 0.54$ & $2.52 \pm 1.81$ & $2.14 \pm 0.10$ & $0.18 \pm 0.01$ & $1.08 \pm 0.05$ \\
\hline & II & $5.40 \pm 1.10$ & $7.46 \pm 0.67$ & $10.19 \pm 0.66$ & $7.68 \pm 1.38$ & $2.44 \pm 0.12$ & $1.82 \pm 0.09$ & $0.84 \pm 0.04$ \\
\hline \multirow[t]{2}{*}{ January 2017} & I & $12.16 \pm 0.53$ & $19.22 \pm 0.66$ & $20.61 \pm 0.63$ & $17.33 \pm 2.61$ & $9.82 \pm 0.47$ & $5.84 \pm 0.28$ & $2.88 \pm 0.14$ \\
\hline & II & $17.72 \pm 085$ & $21.79 \pm 1.36$ & $20.84 \pm 1.33$ & $20.12 \pm 1.22$ & $10.24 \pm 0.49$ & $6.20 \pm 0.30$ & $4.92 \pm 0.24$ \\
\hline \multirow[t]{2}{*}{ February } & I & $26.23 \pm 2.37$ & $27.92 \pm 1.50$ & $31.23 \pm 1.72$ & $28.46 \pm 1.46$ & $14.78 \pm 0.71$ & $7.23 \pm 035$ & $1.32 \pm 0.06$ \\
\hline & II & $35.09 \pm 1.63$ & $37.26 \pm 1.83$ & $38.74 \pm 1.29$ & $37.03 \pm 1.05$ & $16.60 \pm 0.80$ & $9.32 \pm 045$ & $1.08 \pm 0.05$ \\
\hline \multirow[t]{2}{*}{ March } & I & $30.14 \pm 1.95$ & $32.04 \pm 1.17$ & $33.69 \pm 1.04$ & $31.96 \pm 1.02$ & $12.44 \pm 0.60$ & $8.02 \pm 039$ & $0.98 \pm 0.05$ \\
\hline & II & $20.04 \pm 069$ & $20.48 \pm 0.58$ & $22.77 \pm 1.14$ & $21.10 \pm 0.84$ & $9.40 \pm 0.45$ & $6.31 \pm 0.30$ & $0.64 \pm 0.03$ \\
\hline \multirow[t]{2}{*}{ April } & I & $22.04 \pm 1.32$ & $23.87 \pm 1.20$ & $27.28 \pm 1.48$ & $24.40 \pm 1.53$ & $10.34 \pm 0.50$ & $10.21 \pm 0.49$ & $0.92 \pm 0.04$ \\
\hline & II & $15.01 \pm 0.30$ & $18.00 \pm 0.46$ & $20.70 \pm 1.01$ & $17.90 \pm 1.64$ & $10.50 \pm 0.51$ & $6.80 \pm 0.33$ & $1.32 \pm 0.06$ \\
\hline
\end{tabular}

\# Mean \pm SEm; $n=$ Observation of 45 leaflets 
Table.2 Seasonal incidence of Raoiella indica and its natural enemies on areca palms in Sominakoppa, Shivamogga taluk during 2017-18

\begin{tabular}{|c|c|c|c|c|c|c|c|c|}
\hline \multirow[t]{2}{*}{ Month /Year } & \multirow[t]{2}{*}{ Fortnight } & \multicolumn{4}{|c|}{ Mean mite population per $\mathrm{cm}^{2}$ of leaflet } & \multicolumn{3}{|c|}{ Natural enemies per leaflet } \\
\hline & & Top & Middle & Bottom & Mean & $\begin{array}{l}\text { Stethorus } \\
\text { keralicus }\end{array}$ & $\begin{array}{c}\text { Scolothrips } \\
\text { spp }\end{array}$ & $\begin{array}{c}\text { Amblyseius } \\
\text { largoensis }\end{array}$ \\
\hline \multirow[t]{2}{*}{ May 2017} & I & $13.42 \pm 1.25$ & $16.95 \pm 1.22$ & $14.70 \pm 0.96$ & $15.02 \pm 1.03$ & $10.64 \pm 0.41$ & $5.76 \pm 0.24$ & $3.40 \pm 0.14$ \\
\hline & II & $18.29 \pm 1.01$ & $13.13 \pm 1.49$ & $12.29 \pm 1.06$ & $14.57 \pm 1.87$ & $9.26 \pm 0.49$ & $4.56 \pm 0.18$ & $4.18 \pm 0.17$ \\
\hline \multirow[t]{2}{*}{ June } & I & $14.67 \pm 0.66$ & $15.85 \pm 1.38$ & $17.10 \pm 1.01$ & $15.87 \pm 0.70$ & $7.80 \pm 0.36$ & $4.46 \pm 0.30$ & $2.36 \pm 0.20$ \\
\hline & II & $6.90 \pm 0.98$ & $7.82 \pm 0.64$ & $9.07 \pm 0.34$ & $7.93 \pm 0.62$ & $5.80 \pm 0.33$ & $3.58 \pm 0.20$ & $1.76 \pm 0.10$ \\
\hline \multirow[t]{2}{*}{ July } & I & $7.57 \pm 0.51$ & $9.40 \pm 0.51$ & $9.03 \pm 0.82$ & $8.67 \pm 0.55$ & $13.56 \pm 0.53$ & $7.59 \pm 0.39$ & $1.72 \pm 0.07$ \\
\hline & II & $0.00 \pm 0.00$ & $2.20 \pm 0.25$ & $2.80 \pm 0.32$ & $1.67 \pm 0.85$ & $8.76 \pm 0.56$ & $4.47 \pm 0.20$ & $0.48 \pm 0.09$ \\
\hline \multirow[t]{2}{*}{ August } & I & $0.00 \pm 0.00$ & $1.40 \pm 0.49$ & $0.80 \pm 0.27$ & $0.73 \pm 0.40$ & $4.28 \pm 0.27$ & $3.24 \pm 0.13$ & $0.06 \pm 0.00$ \\
\hline & II & $0.23 \pm 0.05$ & $1.64 \pm 0.41$ & $1.93 \pm 0.25$ & $1.27 \pm 0.52$ & $6.20 \pm 0.35$ & $6.98 \pm 0.28$ & $0.08 \pm 0.00$ \\
\hline \multirow[t]{2}{*}{ September } & I & $0.00 \pm 0.00$ & $0.00 \pm 0.00$ & $0.00 \pm 0.00$ & $0.55 \pm 0.00$ & $4.76 \pm 0.19$ & $4.32 \pm 0.17$ & $0.46 \pm 0.02$ \\
\hline & II & $0.00 \pm 0.00$ & $0.89 \pm 0.16$ & $1.26 \pm 0.29$ & $0.72 \pm 0.37$ & $2.84 \pm 0.15$ & $2.20 \pm 0.09$ & $0.64 \pm 0.03$ \\
\hline \multirow[t]{2}{*}{ October } & I & $0.00 \pm 0.00$ & $0.34 \pm 0.18$ & $0.77 \pm 0.08$ & $0.37 \pm 0.22$ & $1.56 \pm 0.08$ & $1.32 \pm 0.09$ & $2.56 \pm 0.12$ \\
\hline & II & $0.00 \pm 0.00$ & $0.40 \pm 0.17$ & $0.12 \pm 0.06$ & $0.17 \pm 0.11$ & $0.71 \pm 0.10$ & $0.80 \pm 0.22$ & $3.28 \pm 0.15$ \\
\hline \multirow[t]{2}{*}{ November } & I & $0.00 \pm 0.00$ & $0.04 \pm 0.04$ & $0.40 \pm 0.15$ & $0.15 \pm 0.12$ & $0.72 \pm 0.03$ & $0.42 \pm 0.14$ & $2.28 \pm 0.21$ \\
\hline & II & $0.00 \pm 0.00$ & $0.00 \pm 0.00$ & $0.23 \pm 0.07$ & $0.08 \pm 0.07$ & $0.48 \pm 0.02$ & $0.40 \pm 0.16$ & $3.16 \pm 0.11$ \\
\hline \multirow[t]{2}{*}{ December } & I & $0.90 \pm 0.29$ & $1.42 \pm 0.41$ & $2.52 \pm 0.40$ & $1.61 \pm 0.47$ & $0.28 \pm 0.08$ & $0.20 \pm 0.06$ & $0.16 \pm 0.07$ \\
\hline & II & $1.02 \pm 0.30$ & $2.20 \pm 0.54$ & $3.65 \pm 0.35$ & $2.29 \pm 0.76$ & $0.28 \pm 0.02$ & $0.10 \pm 0.06$ & $0.80 \pm 0.04$ \\
\hline \multirow[t]{2}{*}{ January 2018} & I & $3.41 \pm 0.42$ & $4.66 \pm 0.85$ & $5.20 \pm 0.57$ & $4.42 \pm 0.53$ & $2.60 \pm 0.18$ & $1.97 \pm 0.17$ & $4.80 \pm 0.24$ \\
\hline & II & $5.12 \pm 0.38$ & $9.18 \pm 1.16$ & $11.45 \pm 0.50$ & $8.58 \pm 1.85$ & $6.20 \pm 0.25$ & $5.84 \pm 0.30$ & $5.20 \pm 0.25$ \\
\hline \multirow[t]{2}{*}{ February } & I & $19.95 \pm 1.12$ & $27.61 \pm 1.49$ & $33.57 \pm 1.09$ & $27.04 \pm 3.94$ & $12.26 \pm 0.70$ & $9.59 \pm 0.50$ & $2.52 \pm 024$ \\
\hline & II & $29.03 \pm 2.05$ & $36.55 \pm 1.93$ & $40.28 \pm 2.49$ & $35.29 \pm 3.30$ & $14.21 \pm 0.68$ & $10.46 \pm 0.57$ & $1.36 \pm 0.12$ \\
\hline \multirow[t]{2}{*}{ March } & I & $47.64 \pm 4.88$ & $49.33 \pm 2.14$ & $43.65 \pm 2.74$ & $46.87 \pm 1.68$ & $16.80 \pm 0.66$ & $12.34 \pm 0.49$ & $1.92 \pm 0.17$ \\
\hline & II & $40.50 \pm 0.93$ & $44.35 \pm 1.60$ & $45.27 \pm 1.88$ & $43.37 \pm 1.46$ & $13.12 \pm 0.58$ & $10.32 \pm 0.41$ & $0.98 \pm 0.25$ \\
\hline \multirow[t]{2}{*}{ April } & I & $29.80 \pm 1.11$ & $30.60 \pm 1.36$ & $32.40 \pm 1.46$ & $30.93 \pm 0.76$ & $12.24 \pm 0.53$ & $9.23 \pm 0.36$ & $0.61 \pm 0.03$ \\
\hline & II & $19.00 \pm 1.05$ & $19.80 \pm 0.35$ & $20.60 \pm 1.21$ & $19.80 \pm 0.46$ & $10.02 \pm 0.55$ & $7.00 \pm 0.39$ & $1.76 \pm 0.08$ \\
\hline
\end{tabular}

\# Mean \pm S. Em ; n= Observation of 45 leaflets. 
Table.3 Seasonal incidence of Raoiella indica and its natural enemies on areca palms in Sominakoppa,

Shivamogga taluk (Pooled data of 2016-17 and 2017-18)

\begin{tabular}{|c|c|c|c|c|c|c|c|c|}
\hline \multirow[t]{2}{*}{ Month / Year } & \multirow[t]{2}{*}{ Fortnight } & \multicolumn{4}{|c|}{ Mean mite population per $\mathrm{cm}^{2}$ of leaflet ${ }^{\#}$} & \multicolumn{3}{|c|}{ Natural enemies per leaflet } \\
\hline & & Top & Middle & Bottom & Mean & $\begin{array}{c}\text { Stethorus } \\
\text { keralicus }\end{array}$ & Scolothrips spp & Amblyseius largoensis \\
\hline \multirow[t]{2}{*}{ May } & I & $14.12 \pm 1.24$ & $16.95 \pm 0.79$ & $17.70 \pm 0.97$ & $16.26 \pm 1.09$ & $13.57 \pm 0.42$ & $8.12 \pm 0.27$ & $3.85 \pm 0.16$ \\
\hline & II & $16.77 \pm 0.87$ & $14.94 \pm 0.63$ & $16.18 \pm 0.64$ & $15.96 \pm 0.53$ & $10.80 \pm 0.42$ & $6.01 \pm 0.21$ & $4.43 \pm 0.19$ \\
\hline \multirow[t]{2}{*}{ June } & I & $14.52 \pm 0.61$ & $14.49 \pm 0.27$ & $18.23 \pm 0.83$ & $15.75 \pm 1.24$ & $8.88 \pm 0.34$ & $5.01 \pm 0.28$ & $1.86 \pm 0.08$ \\
\hline & II & $8.28 \pm 0.60$ & $9.76 \pm 0.60$ & $11.59 \pm 0.17$ & $9.88 \pm 0.95$ & $7.35 \pm 0.29$ & $3.52 \pm 0.18$ & $1.77 \pm 0.07$ \\
\hline \multirow[t]{2}{*}{ July } & I & $6.31 \pm 0.38$ & $7.47 \pm 0.31$ & $9.87 \pm 0.27$ & $7.88 \pm 1.04$ & $9.79 \pm 0.36$ & $4.84 \pm 0.25$ & $1.40 \pm 0.06$ \\
\hline & II & $2.37 \pm 0.71$ & $4.30 \pm 0.38$ & $2.80 \pm 0.24$ & $2.69 \pm 0.58$ & $7.66 \pm 0.39$ & $4.03 \pm 0.18$ & $0.40 \pm 0.05$ \\
\hline \multirow[t]{2}{*}{ August } & I & $2.20 \pm 1.16$ & $3.42 \pm 0.33$ & $3.16 \pm 0.30$ & $2.93 \pm 0.37$ & $4.52 \pm 0.21$ & $3.36 \pm 0.14$ & $0.06 \pm 0.00$ \\
\hline & II & $2.32 \pm 0.20$ & $3.50 \pm 0.25$ & $4.35 \pm 0.29$ & $3.39 \pm 0.58$ & $5.13 \pm 0.26$ & $4.79 \pm 0.19$ & $0.06 \pm 0.00$ \\
\hline \multirow[t]{2}{*}{ September } & I & $1.24 \pm 0.61$ & $2.00 \pm 0.21$ & $1.29 \pm 0.19$ & $1.51 \pm 0.24$ & $3.99 \pm 0.16$ & $3.33 \pm 0.14$ & $0.33 \pm 0.02$ \\
\hline & II & $0.88 \pm 0.21$ & $1.27 \pm 0.16$ & $1.60 \pm 0.12$ & $1.25 \pm 0.20$ & $2.79 \pm 0.14$ & $2.09 \pm 0.09$ & $0.47 \pm 0.02$ \\
\hline \multirow[t]{2}{*}{ October } & I & $0.00 \pm 0.10$ & $3.65 \pm 0.16$ & $4.28 \pm 0.33$ & $2.64 \pm 1.33$ & $3.08 \pm 0.15$ & $1.18 \pm 0.07$ & $1.83 \pm 0.09$ \\
\hline & II & $1.61 \pm 0.88$ & $2.54 \pm 0.23$ & $3.51 \pm 0.38$ & $2.55 \pm 0.54$ & $2.90 \pm 0.16$ & $0.82 \pm 0.09$ & $2.78 \pm 0.13$ \\
\hline \multirow[t]{2}{*}{ November } & I & $0.79 \pm 0.25$ & $1.95 \pm 0.12$ & $2.34 \pm 0.23$ & $1.69 \pm 0.46$ & $1.62 \pm 0.08$ & $0.58 \pm 0.08$ & $2.67 \pm 0.12$ \\
\hline & II & $0.54 \pm 0.20$ & $0.96 \pm 0.26$ & $1.83 \pm 0.17$ & $1.11 \pm 0.37$ & $1.92 \pm 0.09$ & $0.36 \pm 0.07$ & $3.48 \pm 0.14$ \\
\hline \multirow[t]{2}{*}{ December } & I & $0.65 \pm 0.15$ & $1.23 \pm 0.27$ & $4.33 \pm 0.41$ & $2.07 \pm 1.14$ & $1.21 \pm 0.09$ & $0.19 \pm 0.03$ & $0.62 \pm 0.04$ \\
\hline & II & $3.21 \pm 0.66$ & $4.83 \pm 0.50$ & $6.92 \pm 0.44$ & $4.99 \pm 1.07$ & $1.36 \pm 0.07$ & $0.96 \pm 0.07$ & $0.82 \pm 0.04$ \\
\hline \multirow[t]{2}{*}{ January } & I & $7.79 \pm 0.29$ & $11.94 \pm 0.64$ & $12.91 \pm 0.55$ & $10.88 \pm 1.57$ & $6.21 \pm 0.32$ & $5.41 \pm 0.20$ & $3.84 \pm 0.19$ \\
\hline & II & $11.42 \pm 0.45$ & $15.49 \pm 0.51$ & $16.15 \pm 0.74$ & $14.35 \pm 1.47$ & $9.22 \pm 0.35$ & $7.52 \pm 0.29$ & $5.06 \pm 0.24$ \\
\hline \multirow[t]{2}{*}{ February } & I & $23.09 \pm 1.62$ & $27.77 \pm 1.13$ & $32.40 \pm 0.90$ & $27.75 \pm 2.68$ & $19.03 \pm 0.69$ & $12.41 \pm 0.42$ & $1.92 \pm 0.15$ \\
\hline & II & $32.06 \pm 1.56$ & $36.91 \pm 1.73$ & $39.51 \pm 1.74$ & $36.16 \pm 2.18$ & $21.62 \pm 0.60$ & $15.68 \pm 0.41$ & $1.22 \pm 0.08$ \\
\hline \multirow[t]{2}{*}{ March } & I & $38.89 \pm 2.45$ & $40.69 \pm 0.91$ & $38.67 \pm 1.57$ & $39.42 \pm 0.63$ & $21.91 \pm 0.74$ & $17.89 \pm 0.50$ & $1.45 \pm 0.10$ \\
\hline & II & $30.27 \pm 0.58$ & $32.42 \pm 1.00$ & $34.02 \pm 1.04$ & $32.24 \pm 1.08$ & $16.28 \pm 0.50$ & $12.82 \pm 0.33$ & $0.81 \pm 0.13$ \\
\hline \multirow[t]{2}{*}{ April } & I & $25.92 \pm 1.08$ & $27.24 \pm 1.20$ & $29.84 \pm 1.04$ & $27.67 \pm 1.15$ & $18.79 \pm 0.47$ & $14.22 \pm 0.41$ & $0.77 \pm 0.04$ \\
\hline & II & $17.01 \pm 0.51$ & $18.90 \pm 0.22$ & $20.65 \pm 1.01$ & $18.85 \pm 1.05$ & $15.26 \pm 0.47$ & $9.40 \pm 0.36$ & $1.54 \pm 0.07$ \\
\hline
\end{tabular}

\# Mean \pm S. Em ; n= Observation of 45 leaflets. 
Table.4 Correlation and multiple regression coefficient of Raoiella indica and its natural enemies with weather parameters in Sominakoppa, Shivamogga

\begin{tabular}{|c|c|c|c|c|c|c|c|c|}
\hline \multirow[t]{2}{*}{ Year } & \multirow[t]{2}{*}{ Particulars } & \multicolumn{2}{|c|}{$\begin{array}{l}\text { Temperature } \\
\left({ }^{0} \mathrm{C}\right)\end{array}$} & \multicolumn{2}{|c|}{$\begin{array}{l}\text { Relative humidity } \\
(\%)\end{array}$} & \multirow{2}{*}{$\begin{array}{c}\begin{array}{c}\text { Rainfall } \\
(\mathbf{m m})\end{array} \\
\left(\mathbf{X}_{\mathbf{3}}\right)\end{array}$} & \multirow{2}{*}{$\mathbf{R}^{2}$} & \multirow[t]{2}{*}{ Regression Equation } \\
\hline & & $\begin{array}{c}\text { Max } \\
\left(\mathbf{X}_{1}\right)\end{array}$ & $\begin{array}{l}\text { Min } \\
\left(\mathbf{X}_{2}\right)\end{array}$ & $\begin{array}{l}\text { Morning } \\
\qquad\left(\mathbf{X}_{4}\right)\end{array}$ & $\begin{array}{l}\text { Evening } \\
\quad\left(\mathbf{X}_{5}\right)\end{array}$ & & & \\
\hline \multirow[t]{4}{*}{ 2016-17 } & Raoiella indica & $0.498 *$ & -0.007 & -0.330 & $0.528 * *$ & -0.610 & 0.675 & $\begin{array}{c}Y=-44.275+1.868 X_{1^{-}-0.286 X_{2}-0.002 X_{3^{-}}} \\
0.354 X_{4}+0.493 X_{5}\end{array}$ \\
\hline & Stethorus keralicus & $0.519 * *$ & 0.088 & -0.261 & $-0.568 * *$ & -0.019 & 0.717 & $\begin{array}{c}Y=-35.680+1.227 X_{1}+0.004 X_{2}-0.04 X_{3-} \\
0.179 X_{4}+0.315 X_{5}\end{array}$ \\
\hline & Scolothrips spp. & $0.505^{*}$ & -0.022 & -0.266 & $0.576 * *$ & -0.115 & 0.204 & $\begin{array}{c}Y=-33.137+0.987 X_{1}-0.209 X_{2}-0.019 X_{3-} \\
0.087 X_{4}+0.284 X_{5}\end{array}$ \\
\hline & $\begin{array}{l}\text { Amblyseius } \\
\text { largoensis }\end{array}$ & 0.342 & -0.141 & -0.359 & -0.155 & -0.133 & 0.759 & $\begin{array}{c}\mathrm{Y}=3.408+0.136 \mathrm{X}_{1}+0.0204 \mathrm{X}_{2}+0.009 \mathrm{X}_{3}-0.062 \mathrm{X}_{4}- \\
0.016 \mathrm{X}_{5}\end{array}$ \\
\hline \multirow[t]{4}{*}{ 2017-18 } & Raoiella indica & $0.775 * *$ & 0.055 & $-0.777 * *$ & $-0.775 * *$ & -0.306 & 0.759 & $\begin{array}{c}Y=17.249+0.608 X_{1}-0.005 X_{2}+0.207 X_{3^{-}} \\
0.210 X_{4}+0.001 X_{5}\end{array}$ \\
\hline & Stethorus keralicus & $0.721 * *$ & 0.183 & $-0.703 * *$ & $-0.684 * *$ & -0.166 & 0.830 & $\begin{array}{c}Y=5.131+0.984 X_{1}+1.379 X_{2}+0.003 X_{3^{-}} \\
0.735 X_{4}+0.106 X_{5}\end{array}$ \\
\hline & Scolothrips spp & $0.701 * *$ & 0.100 & $-0.761 * *$ & $-0.740 * *$ & -0.208 & 0.831 & $\begin{array}{c}Y=12.021+0.552 \mathrm{X}_{1}+0.963 \mathrm{X}_{2}+0.006 \mathrm{X}_{3^{-}} \\
0.546 \mathrm{X}_{4}+0.059 \mathrm{X}_{5}\end{array}$ \\
\hline & $\begin{array}{l}\text { Amblyseius } \\
\text { largoensis }\end{array}$ & 0.214 & -0.292 & -0.158 & -0.260 & -0.381 & 0.250 & $\begin{array}{c}Y=-5.101+0.086+X_{1}-0.178 X_{2}-0.006 X_{3}- \\
0.155 X_{4}+0.087 X_{5}\end{array}$ \\
\hline \multirow[t]{4}{*}{ Pooled } & Raoiella indica & $0.656 * *$ & 0.026 & $-0.700 * *$ & $-0.468 *$ & -0.254 & 0.808 & $\begin{array}{c}\mathrm{Y}=48.19+1.210 \mathrm{X}_{1}+2.356 \mathrm{X}_{2}-0.012 \mathrm{X}_{3^{-}} \\
1.437 \mathrm{X}_{4}+1.685 \mathrm{X}_{5}\end{array}$ \\
\hline & Stethorus keralicus & $0.636 * *$ & 0.168 & $-0.633 * *$ & -0.319 & -0.116 & 0.845 & $\begin{array}{c}Y=26.52+0.667 X_{1}+1.581 X_{2}+0.006 X_{3-} \\
0.831 X_{4}+9.323 X_{5}\end{array}$ \\
\hline & Scolothrips spp & $0.625 * *$ & 0.059 & $-0.692 * *$ & -0.380 & -0.171 & 0.811 & $\begin{array}{c}Y=23.727+0.513 X_{1}+1.007 X_{2}+0.004 X_{3^{-}} \\
0.645 X_{4}+0.077 X_{5}\end{array}$ \\
\hline & $\begin{array}{l}\text { Amblyseius } \\
\text { largoensis }\end{array}$ & 0.288 & -0.227 & -0.229 & -0.247 & -0.381 & 0.250 & $\begin{array}{c}Y=-5.101+0.086 X_{1}-0.178 X_{2}-0.006 X_{3}+0.155 X_{4^{-}} \\
0.087 X_{5}\end{array}$ \\
\hline
\end{tabular}

** Significance at $\mathrm{p}=0.01$ level; * Significance at $\mathrm{p}=0.05$ level; $\mathrm{n}=24$ 
The lowest of S. keralicus (1.21 / leaflet) and Scolothrips spp. (0.19 / leaflet) were recorded during first fortnight of December. The $A$. largoensis population was lowest during second fortnight of August (0.06 / leaflet), while it attained to maximum density during January, May and November months.

There was a significant positive relationship noticed between maximum temperature $\left(\mathrm{r}=0.620^{* *}\right)$ and mite population, whereas minimum temperature $(\mathrm{r}=0.026)$ had a positive non-significant association (Table 4). Morning relative humidity $(\mathrm{r}=-0.700 * *)$, evening relative humidity $\left(\mathrm{r}=-0.468^{*}\right)$ and rainfall $(\mathrm{r}=-0.306)$ were negatively correlated

S. keralicus had significant positive correlation with maximum temperature $(\mathrm{r}=$ $\left.0.636^{* *}\right)$ whereas, minimum temperature $(\mathrm{r}=$ 0.168) showed positive non-significant association. The morning relative humidity ( $\mathrm{r}$ $\left.=-0.633^{* *}\right)$, evening relative humidity $(\mathrm{r}=-$ $0.319)$ and rainfall $\quad(\mathrm{r}=-0.166)$ exhibited negative relationship.

Similarly, Scolothrips spp. had negative association with morning relative humidity $(\mathrm{r}$ $=-0.692 * *)$, evening relative humidity $(\mathrm{r}=$ 0.380) and rainfall $(\mathrm{r}=-0.171)$ (Table 4). There was positive non-significant relationship observed with minimum temperature $(\mathrm{r}=0.059)$. A. largoensis showed positive correlation with maximum temperature $(\mathrm{r}=0.288)$, while minimum temperature $(\mathrm{r}=-0.227)$, morning relative humidity $(\mathrm{r}=-0.229)$, evening relative humidity $(r=-0.247)$ and rainfall $(r=-0.381)$ had negative relationship (Table 4 ).

The multiple regression value depicted in table 4 indicated that combined and overall impact of all the abiotic factors on mites and natural enemies were to the extent of 25 to 84 per cent respectively.
The decline in mite population with the onset of monsoon and an increase in relative humidity are in conformity with Nair and Daniel (1982). Thus results of our study in all three locations showed that high temperature, low relative humidity and rainfall greatly influenced the incidence of $R$. indica.

Our results are in close conformity with Yadav Babu and Manjunatha (2007) reported that there was a positive correlation observed with mite population and temperature, while relative humidity and rainfall had a negative relationship with mite population.

Similarly, Hoy et al., (2010) reported that, In India, populations of $R$. indica are negatively affected by rainfall and high relative humidity while they are highest during hot, sunny, and dry conditions; Taylor et al., (2011); Prabheena and Ramani (2014) found that $R$. indica densities were significantly higher during hotter and drier periods.

Apart from Abiotic factors, the mite population would also have been checked in the field by natural enemies viz., predatory coccinellid, S. keralicus and predatory thrips Scolothrips spp. During the study period it was observed that their maximum population density coincided with the peak incidence of $R$. indica.

These results clearly indicated that natural enemies are density dependent and had a close association with mite $R$. indica population. Similar results findings were given by Nageshchandra (1980); Somachoudary and Sarkar (1987) who reported association of predatory coccinellid with peak population of $R$. indica are in conformity with present findings.

\section{References}

Hoy, M. A., Pena, J. and Nguyen. R., 2010, Red Palm Mite, Raoiella indica Hirst 
(Arachnida: Acari: Tenuipalpidae). University of Florida IFAS Extension, EENY 397, pp. 1-5.

Nageshachandra, $\quad$ B. $\quad$ K. and Channabasavanna, G. P., 1984, Development and ecology of Raoiella indica Hirst (Acari: Tenuipalpidae) on coconut. Griffiths, D. A. \& Bowman, Acarol., 6(2): 785-790.

Nageshchandra, B. K., 1980, Studies on false spider mites of India, biology and control of Raoiella indica (Acari: Tenuipalpidae) on coconut. Ph.D thesis, Univ. Agric. Sci., Bangalore.

Nair, C. P. K. and Daniel, M., 1982, Pest on arecanut. CPCRI. Kasargud, Kerala. pp. 151-184.

Ochoa, J. J., Beard, G. R., Bauchan, E. C., Kane, A. P. G., Dowling and Erbe, E. F., 2011, Herbivore exploits chink in armor of host. American Entomologist, pp 26-29.

Prabheena, P. and Ramani, N., 2014, Distribution pattern and injurious status of Raoiella indica (Hirst) (Acari:Tenuipalpidae) on arecanut palms. Int. J. Sci and Res. Publications, 4(5): $1-5$.

Somchoudhry, A. K. and Sarkar, P. K., 1987, Observation on natural enemies found in association with coconut mite. Raoiella indica Hirst. Bull. Entomol., New Delhi, 28: 104-107.

Taylor, B., Rahman, P. M., Murphy, S. T. and Sudheendrakumar, V.V., 2011, Withinseason dynamics of red palm mite (Raoiella indica) and phytoseiid predators on two host palm species in south-west India. Exp. Appl. Acarol., pp 15.

Welbourn, C., 2006, Red palm mite Raoiella indica (Acari: Tenuipalpidae). Pest Alert. DPI-FDACS., pp. 4.

Yadav Babu and Manjunatha, 2007, Seasonal incidence of mite population in arecanut. Karnataka J. Agric. Sci., 20(2): 401-402.

\section{How to cite this article:}

Indhusri Chavan, S. Pradeep, M. Manjunatha and H. Narayanaswamy and Sridhara. S. 2020. Seasonal Incidence of Raoiella Indica Hirst and its Natural Enemies on Arecanut. Int.J.Curr.Microbiol.App.Sci. 9(02): 2664-2672. doi: https://doi.org/10.20546/ijcmas.2020.902.303 